\title{
Padres, profesores y pares: contribuciones para la autoestima y coping en los adolescentes
}

\author{
Catarina Pinheiro Mota ${ }^{1 *}$ y Paula Mena Matos ${ }^{2}$ \\ ${ }^{1}$ Universidad de Trás-os-Montes y Alto Douro - Departamento de Educación y Psicologia. Vila Real. Portugal. \\ Centro de Psicología de la Universidad do Porto. Portugal. \\ ${ }^{2}$ Facultad de Psicología y de Ciencias de la Educación de la Universidad do Porto. \\ Centro de Psicología de la Universidad do Porto. Portugal.
}

\begin{abstract}
Resumen: En consonancia con la teoría del apego, la calidad de la relación con figuras significativas juegan un papel clave en el desarrollo psicosocial, contribuyendo, entre otras cosas, para el desarrollo de la autoestima y estrategias de coping. El objetivo de este estudio es determinar la contribución diferencial de variables relacionales (incluidas las relaciones con los padres, pares y profesores) en la predicción de la autoestima y coping en una muestra de 216 adolescentes, entre 14 y 16 años y de ambos sexos. El apego a los padres, las relaciones con los pares y con profesores revelaron diferentes configuraciones de las variables en la explicación de la autoestima y coping. La autoestima es positivamente predicha por la calidad de lazo emocional y negativamente por la inhibición de la exploración y la individualidad en la vinculación a los padres. El coping activo es positivamente predicho por la autoestima y la relación a los pares. Se observó un efecto positivo e indirecto de la calidad del lazo emocional en el coping activo a través de la autoestima y la relación a los pares. La relación con los pares parece ser mejorada por la calidad de la relación con los profesores. Los resultados serán discutidos en función de la teoría del apego y de acuerdo con una perspectiva ecológica del desarrollo.
\end{abstract}

Palabras clave: Variables relacionales; autoestima; coping; adolescencia.

\section{Introducción}

Aún reconociéndose las contribuciones de la teoría del apego en la infancia y más tarde en la edad adulta, la etapa de la adolescencia permanece en un intermedio de vivencia del desenvolvimiento. La adolescencia se encuentra llena de cambios y (re)organizaciones que se van desarrollando en la relación con las figuras cuidadoras primarias y en una perspectiva ecológica y sistémica, la "familia extensa" con los amigos y otros adultos significativos como los profesores en el contexto escolar. El presente estudio tiene como fondo teórico la teoría del apego destacando la importancia de la vivencia emocional en la infancia e adolescencia y su repercusión en las relaciones afectivas posteriores. En una perspectiva ecológica del desarrollo humano, se destaca todavía el role de otras figuras de afecto allá de la familia como son los pares y profesores, fuentes de inevitable aporte de aprendizaje y crecimiento personal.

De este modo, la adolescencia es una etapa que permite expresar las formas de apego seguro proferida por la calidad que ofrece la relación con las figuras parentales. Adolescentes seguros se caracterizan por la capacidad de potenciar y evaluar su relación con las figuras parentales y de forma cordial discutir los puntos de desacuerdo en el contexto de la relación que se caracteriza por la armonía y el apoyo (e.g., Allen

* Dirección para correspondencia [Correspondence address]: Catarina Pinheiro Mota. Universidad de Trás-os-Montes y Alto Douro Departamento de Educación y Psicologia - Edifício do CIFOP - Rua Dr. Manuel Cardona - UTAD, Apartado 1013, 5001-558, Vila Real (Portugal). E-mail: catppmota@utad.pt
Title: Parents, teachers and peers: contributions to self-esteem and coping in adolescents.

Abstract: Consistent with attachment theory, the quality of relationships with significant figures plays a vital role in psychosocial development, contributing, among other aspects, to the development of coping and selfesteem. The purpose of this study is to determine the differential contribution of relational variables (namely relationship with parents, peers and teachers) in predicting coping and self-esteem in a sample of 216 adolescents, 14 to 19 aged, from both genders. Parental attachment, relationships with peers and with teachers evidence different configurations in explaining self-esteem and coping variables. Self-esteem is predicted positively by quality of emotional bond and negatively by parental inhibition of exploration and individuality. Active coping is predicted positively by self-esteem and attachment to peers. However there is an indirect and positive effect of quality of emotional bond in active coping, through self-esteem and peers attachment. Relationship with peers is increased by quality of relationship with teachers. Results are discussed according to attachment theory and an ecological perspective of development.

Key words: Relational variables; self-esteem; coping; adolescence.

et al., 2003; Hershenberg et al., 2011). La adolescencia incorpora también una tarea caracterizada por la necesidad de que los jóvenes se integren en un contexto social donde se incluyen los pares creando un sentimiento de pertenencia (e.g., Cohen, Kasen, Chen, Hartmark \& Gordon, 2003; Eccles, Templeton, Barber \& Stone, 2003; Millings, Buck, Montgomery, Spears, \& Stallard, 2012) Según varios autores, es en el contexto de los pares que se ponen a prueba modos de ser y pensar más diferenciados que recrean y validan el sentimiento de identidad personal (Meeus, Iedema, Maassen \& Engels, 2005). La calidad de las relaciones con los padres y los pares parece comportar impactos distintos en la construcción de las áreas de la identidad, por lo que los compromisos de la escuela tienden a ser resueltos en la relación con los padres, mientras que los compromisos relacionales asumen contornos relevantes en los pares (Meeus, Osterwegel \& Vollebergh, 2002). En este sentido, algunos estudios traducen el efector mediador de los pares en lo que concierne a la asociación de la calidad de la relación hacia los padres y variables de adaptación social. Deković, Wissink y Meijer (2004) en un estudio con 603 adolescentes entre 14 a 16 años, con el objetivo de estudiar el efecto de la calidad de la relación de los padres en el comportamiento social, confirman que los pares pueden ejercer un role mediador potenciando la desadaptación de los jóvenes. Aún, Drew, Berg y Wiebe (2010) en un estudio con 252 jóvenes de 10 a 14 años confirman que la calidad de la relación a los padres potencia el desarrollo de conductas saludables en contextos de enfermedad, más allá del efecto mediador de los pares. Por otra parte, Mota y Matos (2008) en un estudio con 403 adolescentes 
de entre 14 y 19 años de edad destacan el papel mediador positivo de la relación con los pares en la asociación entre la calidad del apego a los padres y el desarrollo de competencias sociales (autocontrol, asertividad y empatía).

Es necesario realzar que el apego aportado con las figuras significativas de afecto, como los padres, traduce el desarrollo de una base segura que a su vez permite a los jóvenes crecer emocionalmente y caminar hacia la calidad de las demás relaciones interpersonales, algunas de ellas también importantes para los jóvenes, recreando verdaderos puertos seguros (e.g., Buhl, 2008; Grossmann \& Grossmann, 2004; Rocha, 2008). Así, también los pares asumen una parte importante en la vida de los adolescentes que es acompañada de cambios (cognitivos, socio emocionales, familiares, vocacionales y de la imagen de sí mismo), desafiando el sentido de la identidad y la autoestima (e.g., Wilkinson, 2010). Estos cambios prepararan a los adolescentes y sus pares para desarrollar la capacidad para convertirse en figuras de apego cuando sean adultos (Freeman \& Brown, 2001; Nickerson \& Nagle, 2005).

Así mismo, la función de la escuela aparece como incuestionable en el contexto del desarrollo, lleno de oportunidades para el crecimiento cognitivo, emocional y social para los jóvenes (e.g. Eccles, \& Roeser, 2003). La relación que los jóvenes tienen con las figuras significativas de la escuela se relaciona con el ajuste personal y académico, asumiendo un papel relevante para el desarrollo psicosocial (e.g., Davis, 2003; Larose, Tarabulsy \& Cyrenne, 2005; Rodas, Grossman \& Resch, 2000; Wentzel, 2002). En consecuencia, este estudio tendrá como base un abordaje de la relación inicial creada con los padres, añadiendo posteriormente la importancia de las relaciones en el contexto de los pares y de los adultos significativos en el contexto escolar, en particular la calidad de la relación con los profesores de la escuela. En Portugal, estudios sobre la calidad de los vínculos con los profesores son escasos, todavía estudios con profesores en asociación con la calidad de la relación con los pares, se revelan un contexto importante de desarrollo psicosocial de los jóvenes. Algunos estudios sugieren que la relación que los jóvenes tienen con las figuras significativas de la escuela se relaciona con el ajuste personal y académico, asumiendo un papel relevante para el desarrollo del bienestar, coping y la autoestima (e.g., AlYagon \& Mikulincer, 2006; Davis, 2003; Soenens \& Vansteenkiste, 2005).

Los profesores pueden desempeñar un papel importante en la forma como los jóvenes eligen e interactúan en diversas situaciones, incluido el grupo de pares. La relación que los jóvenes tienen con la figura del profesor les permite la oportunidad de aprender a "discutir", desarrollando valores, creencias e ideas que observan en sus preferencias sociales y la percepción del propio grupo (e.g., Mikami, Gregory, Allen, Pianta, \& Lun, 2011). Por lo tanto, las representaciones que los jóvenes crean de sí mismos parecen relacionarse con la imagen de sí devuelta por los adultos. La figura del profesor ha adquirido una posición dominante en su función de apego, aunque no sea considerada en el mismo plano que la na- turaleza de la relación establecida con los padres (e.g., Matos, 2003; Zimmer-Gembeck \& Locke, 2007). Wentzel (2002) sugiere que el modelo puede ser generalizado a contextos externos, incluida la identificación de las relaciones con los profesores, con una promoción de la comunicación, control, madurez, fomentando la motivación y adaptación a la escuela.

A lo largo de este estudio existe además una preocupación de examinar en qué medida la calidad de las relaciones potencia la adaptación psicosocial de los adolescentes. Todavía, este es un concepto muy amplio, por lo que hemos decidido analizar algunas variables que podrían representar de forma verosímil y viable el concepto de adaptación de los jóvenes. El coping y la autoestima son las variables elegidas por ser generales y multidimensionales, capaces de aportar elementos sociales, cognitivos y emocionales, pero al mismo tiempo bastante representativas en lo que concierne al sentido de adaptación psicosocial.

\section{Implicaciones de la calidad de las relaciones en la autoestima y coping}

Originalmente Bowlby (1969, 1973) teoriza que el sistema del apego se activa por estímulos ambientales del estrés en los niños que buscan la proximidad con sus cuidadores. La falta de respuesta de los cuidadores parece influir no solo en el comportamiento inmediato de los niños (Ainsworth, Blehar, Waters \& Wall, 1978), como también en las relaciones interpersonales futuras en la adolescencia y la edad adulta. Por lo tanto, es claro que el coping surge en el contexto de la calidad de las relaciones con las figuras significativas que, en una perspectiva de aprendizaje social, permiten que los jóvenes aprendan a hacer frente a sus inquietudes.

Es importante destacar que la escuela tiene un papel relevante en el desarrollo de los jóvenes, no agotándose en los aspectos de naturaleza académica, aportando diversas áreas de la esfera humana, incluyendo las dimensiones de la calidad de la relación, competencia personal y la construcción de la identidad, que por supuesto es compartida con el papel de la familia (Matos, 2003). De encuentro al punto de vista ecológico, el desarrollo de los jóvenes en la escuela se asocia con el mayor número de interacciones significativas establecidos entre los participantes de este contexto (que incluye los pares, profesores, funcionarios de las escuelas y otras figuras significativas), así como la calidad de estas relaciones, lo que sugiere una mayor posibilidad de enriquecimiento y crecimiento interpersonal (Bronfenbrenner, 1996).

De este modo, contrariando un proyecto social y cultural que tiende a centrarse en el individualismo y el espíritu de la competencia, se pretende entender la escuela más allá de un conjunto de conocimientos disciplinares y las competencias cognitivas, dando importancia al establecimiento de la relación. El contexto de base segura potencia el aprendizaje de las relaciones, inicialmente con los objetos físicos y, a continuación, con los objetivos sociales, incluyendo el contacto con pares y adultos significativos (Matos, 2003). La relación 
es, por tanto, un factor importante en el aprendizaje, porque los jóvenes aprenden a expresar y regular las emociones. La escuela promueve el desarrollo de los recursos emocionales sobre todo por el apoyo de los profesores y los compañeros, aumentando la autoestima, motivación, empatía y estrategias constructivas para resolver los conflictos internos y externos (e.g., Pianta, 1999; Riley, 2011; Zimmer-Gembeck \& Locke, 2007).

La autoestima es un indicador de la calidad relacional en las primeras fases de la vida, ya que la consideración positiva de si, fruto de las relaciones próximas y disponibles de los padres, potencia jóvenes con más confianza, y por lo tanto, más capaces de volverse para el exterior, experimentando el pedir ayuda, desarrollando así un coping más activo (e.g. Arndt \& Goldenberg, 2002; Assor \& Tal, 2012). Según este punto de vista, cuando hay altos niveles de estrés, los jóvenes con alta autoestima muestran una mayor voluntad de buscar y gestionar estrategias para la resolución de dificultades. Algunos estudios han descrito la autoestima como estado significativamente asociado con el bienestar subjetivo y la satisfacción en las relaciones sociales (e.g., Leary \& Baumeister, 2000). Rocha (2008) en un estudio de adolescentes y jóvenes adultos, encuentra una estrecha relación entre la autoestima y la percepción de la aceptación de amigos y familiares, asimismo la percepción clara de esta aceptación tiende a aumentar la auto-estima.

Así, la percepción de una gran aceptación y la autoestima parecen relacionase a una vinculación segura, lo que confirma la hipótesis de que los modelos de vinculación influyen en la evaluación global del self. Varios autores (e.g., Laible, Carlo \& Roesch, 2004; McLean \& Jennings, 2012) afirman que la autoestima parece tener un efecto positivo en la integración en la comunidad social en general (que incluye no sólo la familia, sino también el grupo de pares y el contexto escolar). Jiménez (2011) acrece en su estudio con 414 adolescentes que la autoestima ejerce un papel mediador significativo en la influencia de la calidad del clima familiar y en consumo de sustancias de los adolescentes, por lo que se concluye que parece necesario considerar la relación familiar como un antecedente relevante de la autovaloración de los adolescentes. Un contexto relacional de seguridad y estabilidad es un recurso para el aprendizaje conduciendo a una posibilidad de exploración del mundo exterior. La seguridad aportada a esta exploración facilita la relación con los demás, proporcionando un importante indicador de la madurez emocional (Dubois, Felner, Marca, Adan \& Evans, 1992). Por lo tanto, la relación afectiva con el profesor representa un factor clave para el aprendizaje, la calidad del aprendizaje también parece traer contribuciones significativas a la mayor representación de sí mismo y de los demás, fomentando la adaptación al contexto escolar (e.g., Zimmer-Gembeck \& Locke, 2007). El mayor o menor éxito en el desarrollo del aprendizaje del joven, requiere un contexto relacional de apoyo, capaz de potenciar la autoestima y prevenir sentimientos de culpa e inadecuación personal.

\section{Método}

\section{Objetivos e hipótesis}

Según el modelo conceptual en hipótesis se pretende observar el efecto del apego parental y de la relación con los profesores en el coping y la autoestima de los adolescentes. Al mismo tiempo, se pretende analizar si el apego parental y la relación con los profesores predicen la calidad de las relaciones con los pares. Teniendo en cuenta los conceptos teóricos se espera observar los efectos directos de la relación con los pares en el coping y la autoestima. Por último se considera la hipótesis de existencia de un efecto de mediación de la calidad de la relación con los pares en la asociación entre el apego a los padres y el coping y la autoestima. El modelo conceptual que discrimina las hipótesis es sistematizado en la Figura 1 .

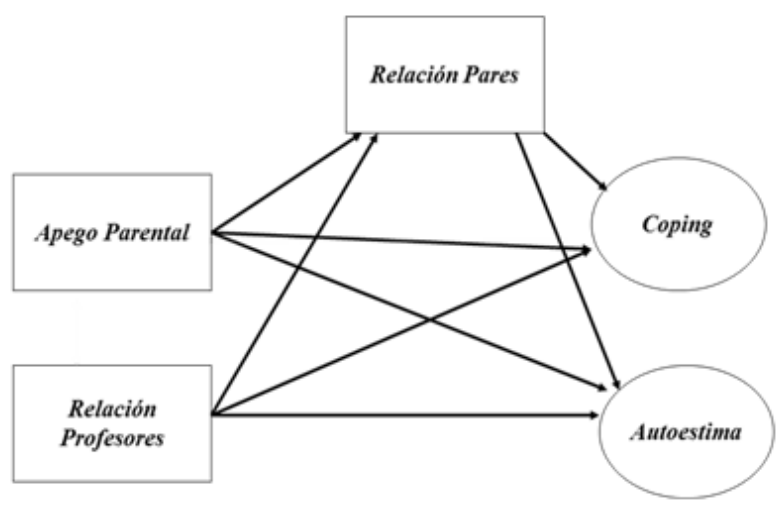

Figura 1. Modelo conceptual del estudio.

\section{Participantes}

El estudio consta de 216 adolescentes que asisten la escuela secundaria $\left(M_{\text {escolaridad }}=10.25, D T=.97\right)$, con edades comprendidas entre los 14 y 19 años $(M=16.30, D T=$ 1.21), $118(54.6 \%)$ de sexo femenino y $98(45.4 \%)$ de sexo masculino. Del total de participantes, $115(53.6 \%)$ procedían de familias intactas y 101 (46.4\%) de familias divorciadas. La edad de los padres varía entre 34 y 85 años $(M=45.31$, DT $=5.8)$ con una media de 7.22 años de escolaridad $(D T=$ $.65)$, la edad de las madres varía entre el 29 y 56 años $(M=$ $42.30, D T=4.8)$ con un promedio de escolaridad de 7.74 años $(D T=.90)$.

\section{Instrumentos}

Todos los instrumentos fueron sometidos a análisis de las propriedades psicométricas, incluida la consistencia interna y análisis factorial confirmatorio, mostrando índices de ajuste en conformidad con los valores críticos, respectivamente, más de .90 por $A G F I, G F I, C F I$ y valores por debajo de .080 para el RMR y RMSEA (Bollen, 1990; MacCallum, Widaman, Preacher \& Hong, 2001; Yuan, 2005). 
Cuestionario de Apego al Padre y la Madre - CAPM (Matos \& Costa, 2001; Gouveia \& Matos, 2012).- Se trata de un cuestionario constituido en su forma revisada por 30 ítems que reflejan las declaraciones acerca de las relaciones familiares. Trata de evaluar las representaciones del apego de los adolescentes y jóvenes adultos a los padres, centrándose en tres dimensiones: la inhibición de la exploración y la individualidad (iei) ("Mis padres siempre interfieren en mis asuntos"), la calidad del lazo emocional (cle) ("Tengo confianza que mi relación con mis padres va a mantenerse en el tiempo"), y por último, la ansiedad de separación (as) ("Es fundamental para mi que mis padres estén de acuerdo con todo lo que pienso"). El formato de la respuesta implica una escala tipo Likert de seis puntos, que se lleva a cabo para el padre y la madre, respectivamente. Los alfas de Cronbach para esta muestra son: iei padre $=.81$, iei madre $=.82$; cle padre $=$. 94 ; cle madre $=.92$; as padre $=.85$; as madre $=.83$. El análisis factorial confirmatorio muestra adecuados índices de ajuste para el modelo (RMR padre / madre $=.068 / .047$; GFI padre / madre $=.964 / .967 ; A G F I$ padre / madre $=$ $.922 / .927 ;$ CFI padre/madre $=.979 / .975 ;$ RMSE $A$ padre / madre $=.072 / .074$ ).

Inventario de Apego a los Padres y Pares - LAPP (Armsden \& Greenberg, 1987; adaptación de Ferreira \& Costa, 1998).Sólo se utilizó la versión de pares, que consta de 25 ítems con una distribución en 3 dimensiones: confianza ("Mis amigos me comprenden"), comunicación ("Me gusta tener la opinión de mis amigos en los asuntos que me preocupan"), $y$ alienación ("Hablar de mis problemas con mis amigos hace que me sienta avergonzado"). El formato de respuesta es tipo Likert con 6 puntos. Los alfas de Cronbach para esta muestra son: confianza $=.86$; comunicación $=.85$ y alienación $=$ .82. El análisis factorial confirmatorio muestra adecuados índices de ajuste del modelo $(R M R=.029, G F I=.967, A G F I$ $=.929, C F I=.975$, RMSE $A=.074)$.

Cuestionario de Relación con Profesores y Funcionarios - CRPF (Mota \& Matos, 2005).- Se trata de un cuestionario de autoreporte construido en la secuencia de la dificultad en encontrar un instrumento capaz de evaluar la calidad de la relación emocional con las figuras significativas, más allá de las figuras parentales. Por lo tanto, el cuestionario trata de evaluar la percepción de la importancia de la relación con profesores y funcionarios de la escuela. Se construyó una versión original que consta de 12 ítems distribuidos en dos dimensiones - profesores: "Me siento triste cuando un profesor se enfada conmigo" y funcionarios de la escuela: "Creo que algunos de los funcionarios de la escuela están disponibles para ayudarme". Cada ítem utiliza una escala de respuesta tipo Likert con seis alternativas de respuesta, de "totalmente en desacuerdo" a "totalmente de acuerdo". El alfa de Cronbach es de .85 para los funcionarios de la escuela, y .75 para los profesores. El análisis factorial exploratorio de rotación varimax con 2 componentes principales reveló que los ítems se saturan en los factores propuestos inicialmente durante la construcción del instrumento (Test-Kaiser-Meyer-Olkin KMO - .879; Barlett Test - 1188.73, $\mathrm{p}=.000)$, constándose $25.6 \%$ y $20.3 \%$ de la varianza explicada para la dimensión de los funcionarios de la escuela y profesores, respectivamente.

Escala de Autoestima de Rosenberg (Rosenberg, 1965; Adaptación de Rocha \& Matos, 2003).- Esta es una escala que consta de 10 ítems destinados a la evaluación de la autoestima en los adolescentes, mediante una escala tipo Likert de 6 puntos de evaluación cuantitativa ("Siento que tengo buenas cualidades"). El alfa de Cronbach para esta muestra es de 86. Un análisis factorial confirmatorio muestra adecuados índices de ajuste $(R M R=.029$ de $G F I=.952, A G F I=.914, C F I$ $=.963, \mathrm{RMSE} A=.072)$.

Coping Across Situations Questionnaire - CASQ (SeiffgeKrenke, 1995; Adaptado por Cleto \& Costa, 1995).- Pretende evaluar las estrategias de coping frente a situaciones adversas, caracterizando el modo como los adolescentes se enfrentan con los agentes de estrés cotidiano. Está organizado de acuerdo a una matriz bidimensional que incluye 20 estrategias de coping aplicadas a 8 áreas problemáticas especificas de la adolescencia: estudios, profesores, padres, pares, relaciones amorosas, self, futuro y tiempo libre. El CASQ se compone de 3 dimensiones de coping: (1) Coping activo (7 ítems), que incluye acciones tales como la búsqueda activa de información y movilización de recursos sociales ("Hablo de los problemas tan pronto como aparecen y no me preocupo más" - ítem 2), (2) Coping interno (6 ítems), que enfatiza la evaluación de la situación de los adolescentes y la necesidad de buscar un compromiso ("Acepto mis límites" - ítem 5), (3) Coping retractivo/evitativo (7 ítems), que implica un enfoque fatalista de las situaciones, que da lugar a un alejamiento e/o negación y la incapacidad para resolver problemas ("Espero lo peor" - ítem 4). El alfa de Cronbach para esta muestra es: coping activo .69; coping interno .57 y coping retractivo .69. Un análisis factorial confirmatorio muestra índices de ajuste aceptables: $R M R=.161 ; C F I=.836 ; R M S E A$ $=.070$. Este modelo es representativo de la estructura teorica más competitiva (para más detalles ver Mota \& Matos, 2006), y es consistente con los resultados y discussiones aportadas por los autores originales (Seiffge-Krenke, 1995; Seiffge-Krenke \& Shulman, 1990).

Cada ítem anotado cuenta como un punto, sumándose en el final para todas las situaciones problemáticas, por lo que se obtiene la frecuencia que utiliza esa estrategia específica de coping. El número más alto alcanzado en todas las estrategias incluye la dimensión de coping más utilizada. El coping activo y el coping interno son considerados estilos funcionales de coping, al contrario del coping retractivo considerado como un estilo de coping disfuncional.

También se utilizó un cuestionario demográfico, que incluía información sobre el sexo, la edad, la identificación de la familia, tal como la edad, profesión, escolaridad y estado civil de las figuras parentales; la identificación escolar y los 
proyectos futuros, así como información sobre la cuestión del divorcio.

\section{Procedimiento}

Tratándose de un estudio transversal, la recogida de datos se realizó en un solo momento en las escuelas secundarias públicas del norte y centro de Portugal escogidas de forma aleatoria. La aplicación se llevó a cabo en horario escolar con el apoyo del equipo de investigación durante 6 meses. Durante la misma se presentaron los objetivos generales del estudio, por lo que, tratándose de una administración colectiva, se aportaron instrucciones estándar de esclarecimiento en lo que respecta a la respuesta a los cuestionarios de auto-informe, destacando en todo momento la confidencialidad de la información inherente a los cuestionarios, y el carácter voluntario de la participación en el estudio. El orden de los cuestionarios fue invertido aleatoriamente para evitar la inverosimilitud de los resultados derivados de la fatiga y el factor de la secuencia de los instrumentos. Del punto de vista ético fue obtenido consentimiento del director de la escuela y de los jóvenes, garantiéndose también el consentimiento informado de los tutores escolares de los jóvenes para la participación en la investigación.

\section{Análisis de datos}

Los datos fueron analizados de acuerdo con un modelo de ecuaciones estructurales - MEE (Original SEM- Structural Equation Modeling, a través del programa EQS, versión 6.1). Este análisis combina en sí mismo la construcción del análisis factorial, análisis de caminos (path analyses) y de regresión, que comprende las relaciones de causalidad y la interdependencia entre las variables, dividiendo los efectos totales en efectos directos e indirectos y testando los índices de ajuste en el modelo global (Shipley, 1999). Como una técnica de confirmación más que de exploración, se pretendió determinar la validez del modelo teórico construido en este estudio. El modelo muestra cómo las variables relacionales influyen entre si y predicen el coping y la autoestima en los adolescentes.

Se realizo la limpieza de la muestra excluyendo cuestionarios incompletos o inadecuados. Se aseguró la retirada de cuestionarios con más del 10\% de abstenciones de respuesta (missings). Un procedimiento fue desarrollado con el fin de analizar los valores extremos (outliers) cuyas respuestas podrían estar lejos de la media, y por lo tanto afectar a la media y la desviación estándar de la muestra (Field, 2005). Por lo tanto, se procedió a identificar valores atípicos multivariados mediante el análisis de la distancia de Mahalanobis, ya que permite el uso de los grupos de media y la varianza para cada variable, por lo que elementos con valores críticos fueron eliminados por apartarse significativamente de la media global de la muestra (Field, 2005).

Se utilizó el método de máxima verosimilitud, habiendo sido anteriormente testada la normalidad de la muestra en es- tudio. Para ello se procedió a realizar e interpretar la prueba de Kolmogorov-Smirnov para muestras grandes con el fin de analizar el valor de significación (p), así como las gráficas de histogramas, gráficos QQ y diagramas de dispersión desde Boxplots que proporcionan información sobre la distribución de los datos (Pallant, 2001). También se calcularon las medidas de asimetría (skeeweness) y plenitud (curtosis) de los elementos de datos de la muestra alrededor de la media, obteniéndose normalidad con valores dentro del rango de la unidad (-1 y 1) (Moroco, 2007). De acuerdo con los valores calculados, se encontró que la muestra cumple los criterios de inclusión de la normalidad por lo tanto, se procedió al análisis estadístico.

Cada una de las variables relacionales se introdujo inicialmente en función a las dimensiones originales de cada uno de los instrumentos con el fin de entender las relaciones que tienen sentido de acuerdo con las predicciones teóricas, y si la presencia de estas variables estaría ajustada para atender a las cuestiones principales en este estudio. En este sentido, las variables que entraron en el modelo inicial fueron el apego a los padres (con las dimensiones de la inhibición de la exploración y la individualidad, la calidad del lazo emocional y la ansiedad de separación, que es el resultado de la agregación de las variables del padre y la madre). Este cálculo es realizado de acuerdo con la media de los valores de padre e madre, teniendo en cuenta que se trata de un cuestionario robusto e cuya consistencia interna de padre e madre juntos es adecuada. También se incluyo la relación con los pares (con dimensiones de la comunicación, alienación y confianza), y finalmente la relación en la escuela (con las dimensiones de la calidad de relación a los profesores).

No obstante, algunas de las dimensiones del modelo se han eliminado, incluyendo la ansiedad de separación en el apego a los padres, la dimensión de alienación, en la relación a los pares. La decisión de eliminar estas variables se relaciona con el hecho de que no se encuentran suficientemente correlacionadas con otras variables de la dimensión, no garantizando que todas las variables midieran un solo contenido teórico. Por otra parte, la presencia de estas dimensiones incluidas en las respectivas variables impidió el logro de índices adecuados de ajuste, que permitan comprender la importancia de las variables relacionales en la autoestima e coping. Es relevante tener en cuenta que ambas variables se correlacionaron negativamente con el resto de variables. Para analizar el impacto diferencial de los contextos relacionales, en cada una de las variables predictas, el coping activo (ya que las otras dimensiones del coping no garantizan la medida del contenido teórico) y la autoestima se presentaron por separado, teniendo en cuenta sólo un indicador de acuerdo con la propuesta de Little, Cunningham, Shahar y Widman (2002).

Téngase en cuenta que este modelo contiene en sí una combinación de modelos de medición, con la formación de medidas de agregación (Bandalos \& Finney, 2001), para la elaboración de las dimensiones de cada variable (como las dimensiones de la calidad del lazo emocional y la inhibición de la exploración y la individualidad para la variable de apego 
a los padres) y los modelos estructurales que correlacionan variables latentes de forma unidireccional y bidireccional. El análisis de acuerdo con el test de Sobel (Baron \& Kenny, 1986) ha observado la existencia de efectos mediadores de la calidad del apego a los padres para el coping activo a través de la calidad de relación con pares y la autoestima (zpares $=1,98$; $S E=.017 ; p=.046 ; z_{\text {autoestima }}=2,12 ; S E=.018 ; p=.033$ ).

Analizando la descomposición de los efectos (por el valor de $\beta$ estandardizado, el error en su valor no estandardizado y la probabilidad con significancia de 0.5 ) se constató además de la existencia de la mediación, la presencia de efectos indirectos (Preacher \& Hayes, 2004), tal como se presenta en la Figura 2.

\section{Datos preliminares}

Tabla 1. Correlación entre las dimensiones en estudio.

\begin{tabular}{|c|c|c|c|c|c|c|c|}
\hline & APEGO & MADRE/PADRE & QLPF & AUTOESTIMA & PARES & & COPING \\
\hline & IEI & CLE & Profesores & & Comunicación & Confianza & Activo \\
\hline \multicolumn{8}{|l|}{ APEGO MADRE/ } \\
\hline PADRE & 1 & $-.366 * *$ & .037 & $-396 * *$ & .025 & -.085 & $-169 *$ \\
\hline IEI & & 1 & .085 & $.333 * *$ & $.161 *$ & $.203 * *$ & $.199 * *$ \\
\hline \multicolumn{8}{|l|}{ CLE } \\
\hline \multicolumn{8}{|l|}{$Q L P F$} \\
\hline Profesores & & & 1 & .005 & $.282 * *$ & $.211 * *$ & $.209 * *$ \\
\hline AUTOESTIMA & & & & 1 & .119 & $.150 *$ & $.187 * *$ \\
\hline \multicolumn{8}{|l|}{ IPPA - PARES } \\
\hline \multirow[t]{2}{*}{ Comunicación } & & & & & 1 & $.762 * *$ & $.217 * *$ \\
\hline & & & & & & 1 & $.191 * *$ \\
\hline Confianza & & & & & & & \\
\hline COPING Activo & & & & & & & 1 \\
\hline
\end{tabular}

La Tabla 1 representa el resultado de las correlaciones entre las variables en estudio. Podemos observar que las dimensiones de calidad del apego a madre y padre presentan una correlación de la inhibición de la exploración y individualidad (IEI) negativa con la autoestima (-.396**) y el coping activo $\left(-.169^{*}\right)$. Por otra parte la dimensión de la calidad del lazo emocional con la madre y padre presentan correlaciones positivas con la comunicación $\left(.161^{*}\right)$ y la confianza $\left(.203^{* *}\right)$ con los pares amigos, a la vez que presenta una correlación positiva con la autoestima $\left(.333^{*}\right)$ y el coping activo (.199**).

En lo que concierne a la relación con los profesores observamos que presentan una correlación positiva con la comunicación $\left(.282^{* *}\right)$ y confianza $\left(.211^{* *}\right)$, a los pares además de una correlación positiva con el coping activo (.209**). La confianza con los pares presenta una correlación positiva con la autoestima $\left(.150^{*}\right)$ y el coping activo (.191**), mientras la comunicación apenas presenta una correlación positiva con el coping activo (.217**). Por último destacamos que la autoestima se correlaciona positivamente con el coping activo $\left(.187^{* *}\right)$ (Tabla 1).

APEGO MADRE/PADRE: IEI- INIBICIÓN DE LA ESPLORACIÓN Y LA INDIVIDUALIDAD: CLE- CALIDAD DEL LAZO EMOCIONAL

La Tabla 2 representa la media y el desvío padrón de cada una de las variables en estudio. Todas las variables se encuentran en un mínimo de 1 y máximo de 6 , á excepción del coping (entre 0 e 2). El resultado del coping activo es el más bajo (1.35) a la vez que los demás resultados se encuentran en un valor medio de 3.95 y 4.81 (Tabla 2).

Tabla 2. Media y Desvío Padrón de las variables en estudio.

\begin{tabular}{|c|c|c|c|c|c|c|c|}
\hline & APEGO & MADRE/PADRE & QLPF & AUTOESTIMA & & PARES & COPING \\
\hline & IEI & CLE & Profesores & & Comunicación & Confianza & Activo \\
\hline Medias & 3.18 & 4.75 & 3.95 & 4.33 & 4.75 & 4.81 & 1.35 \\
\hline Desvio Padrón & .89 & .99 & .95 & .82 & .64 & .58 & 1.1 \\
\hline Minimo & 1 & 1 & 1 & 1 & 1 & 1 & 0 \\
\hline Máximo & 6 & 6 & 6 & 6 & 6 & 6 & 2 \\
\hline
\end{tabular}




\section{Resultados}

El modelo (Figura 2) presentó índices de ajuste dentro de los valores críticos, el GFI, $A G F I$ y $C F I$ por encima de .90 , y índices de RMR y RMSEA con valores por debajo de .080 (Byrne, 1998). La magnitud de los valores observados, se describe a continuación, y constituyen los valores de $\beta$ significativos y estandarizados.

Analizando los resultados (Figura 2) se observó que, tal como se esperaba, la autoestima es predicha de forma positiva por la calidad del lazo emocional $(\beta=.23)$, y de forma negativa por la inhibición de la exploración y la individualidad $(\beta=-.30)$, en el apego a los padres. Todavía se señala que la calidad del lazo emocional tiene un efecto positivo en la confianza y la comunicación en la relación con los pares $(\beta$ $=.19)$. Así pues, la relación con los padres asume un papel importante para los adolescentes ya que les aporta una base segura que se traduce en la posibilidad de abertura hacia el exterior, especialmente en la exploración de nuevas relaciones con sus compañeros. En este sentido, la relación con los pares parece ser de extrema importancia en la organización interna de los adolescentes. Aunque diferente del apego a los padres, esta relación es importante en el desarrollo personal de los adolescentes, representando oportunidades para el desarrollo de competencias de comunicación y los sentimientos de confianza en si mismo y en los demás en una relación horizontal.

Por otra parte, el coping activo, visto como una estrategia de adaptación, que se refiere a la demanda de ayuda activa, es predicho por la relación con los pares $(\beta=.20)$ y la autoestima $(\beta=$.15). Los adolescentes con mayor autoestima y una mejor comunicación y confianza en las relaciones con sus compañeros parecen desarrollar estrategias para buscar ayuda sin miedo a ser rechazados. Cabe señalar todavía, que es posible observar una mediación total de la calidad de las relaciones con los pares $(z=1,98 ; S E=.017 ; p=.046)$ y la autoestima $(z=2,12 ; S E=.018 ; p=.033)$ en la asociación entre el apego a los padres y el coping. A saber, la calidad del lazo emocional tiene un efecto indirecto en el coping activo a través de la mediación de la relación con los pares $(\beta=.38)$, y la autoestima $(\beta=.35)$.

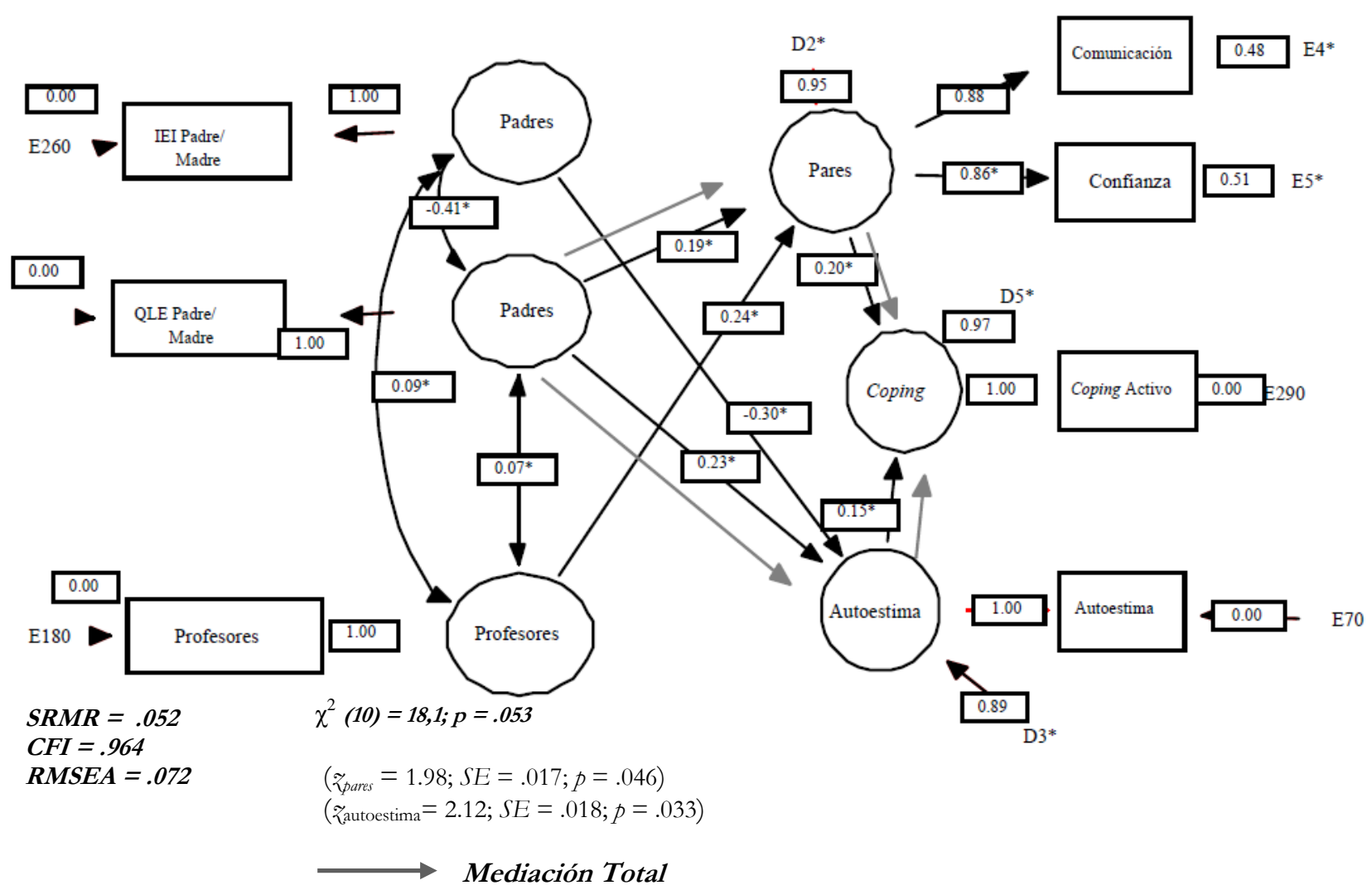

Figura 2. Modelo representativo de la influencia del apego a los padres, la relación a los pares y profesores en el coping activo y autoestima de los adolescentes.

El modelo muestra también que la calidad de la relación con los profesores predice la calidad de las relaciones con los pares $(\beta=.24)$. Este resultado se muestra de acuerdo con lo esperado ya que la relación con el profesor desempeña una elemental fuente de apoyo emocional, ayudando a superar las situaciones de riesgo y potenciando el crecimiento interno, 
incluida la relación con si mismos y con los otros. El modelo presenta valores de ajustamiento adecuado y índices de bondad robustos $\left[\chi^{2}(10)=18,1 ; p=.053 ; C F I=.964 ; \mathrm{SRMR}=\right.$ .052; RMSE $A=.072]$, tal como se observa en la Figura 2.

\section{Discusión}

A pesar de que la literatura ha venido enfatizando a lo largo del tiempo el papel de las figuras parentales como una base segura para el desarrollo de las variables de adaptación psicosocial de los adolescentes, actualmente muchos estudios asumen una perspectiva ecológica sobre la importancia de la diversidad y la calidad de las relaciones en la adolescencia. Así mismo, es inevitable para entender el desarrollo personal de los jóvenes la interacción con el mundo exterior, ya que desde sus inicios los niños mantienen un estrecho contacto con los contextos más allá de la familia. La entrada en la escuela es, quizás, el ejemplo más claro de esta interacción, reportando la ejecución de las tareas esenciales para el crecimiento de los niños, en una combinación de preparación cognitiva y emocional para el futuro. Hablamos principalmente de la carga emocional en cada una de las interacciones en la escuela con los pares y profesores. El presente estudio trató de discutir el papel que estas figuras ejercen en un complemento de figuras parentales, a menudo como un vehículo intermediario en el desarrollo personal de los jóvenes. Los resultados descritos en este estudio se conducen de acuerdo con esta perspectiva, por lo que confirman las hipótesis planteadas.

La calidad de la relación desarrollada con los padres juega un papel importante en el desarrollo de la autoestima de los jóvenes (positivo en la calidad del lazo emocional y negativo en la inhibición de la exploración y la individualidad), por lo que es un significativo preeditor de una base segura tal como refieren varios estudios en este dominio (e.g., Engels, Dekovic \& Meeus, 2002; Mota \& Matos, 2009; Robins \& Trzesniewski, 2005). Guedeney y Guedeney (2004) sugieren que el apego parental potencia la exploración en la adolescencia, desarrollando la reciprocidad, la similitud y el intercambio de experiencias en la relación con los pares. Esta cuestión también aparece en los resultados observados en la medida que la calidad del lazo emocional a los padres predice la calidad de la relación a los pares. Contrariamente al esperado el apego a los padres no predice el coping de forma directa, por lo que se concluye que pueden existir otras variables capaces de ejercer un role significativo para el desarrollo emocional de los jóvenes y su capacidad de hacer frente a las dificultades.

Pero incluso en una perspectiva ecológica, Pianta, Steinberg y Rollins (1995) ponen en relieve el papel de los profesores como una importante fuente de apoyo emocional, especialmente para los adolescentes, ayudándoles a atravesar las situaciones de riesgo y a desarrollar un sentido integrativo del self. La calidad de la relación con los profesores parece asumir un significativo apoyo para expresar y regular las emociones en el contexto escolar, especialmente en la relación con los pares (e.g. Mikami, Gregory, Allen, Pianta, \&
Lun, 2011; Pianta, 1999; Verschueren, Doumen, \& Buyse, 2012). A su vez la relación que tienen los adolescentes con sus pares parece ejercer efectos significativos en el desarrollo psicosocial, y en este estudio particularmente en el coping activo. En esta medida la sensación de ser aceptado por los profesores y los pares permite la construcción de sentido de pertenencia y valor personal, que parece ser relevante en la comprensión de la adaptación psicosocial de variables como la autoestima y coping (e.g. Beyers \& Seiffge-Krenke, 2007; Gifford-Smith \& Brownell, 2003; Llinares, Molpeceres, \& Musitu, 2001). Nótese que en el presente estudio la relación con los pares no predice la autoestima, facto no esperado, aún que la literatura no sea unánime en lo que toca al role de los pares en el desarrollo del bien-estar de los jóvenes (Benson, McWey, \& Ross, 2006). En el presente estudio pensamos que el apego a los padres constituye una variable más significativa en lo que concierne a la predicción de la autoestima de los jóvenes, por lo que el efecto de los pares pude estar camuflado.

Finalmente un aspecto importante observado en este estudio es el efecto positivo que ejerce la autoestima en el coping activo. Es necesario tener en cuenta que una alta autoestima en los adolescentes promueve una mayor adaptación social y una mayor facilidad en el establecimiento de relaciones con calidad. Así pues, los adolescentes con mayor autoestima parecen tener una mayor seguridad que les permite buscar más activamente el apoyo de otros para aprender a gestionar situaciones de estrés (Arndt, \& Goldenberg, 2002).

Particularmente en el presente estudio se observó un resultado interesante que refleja la mediación total de las variables de "relación con sus pares - amigos" y la "autoestima" entre la asociación de la calidad del apego a los padres y el coping. Esto implica que un alto apego con los padres aumenta la calidad de la comunicación y la confianza en la relación con sus pares, así como la autoestima, hecho que promueve el desarrollo de una búsqueda activa de apoyo. Estudios corroboran esta idea, por lo que la calidad del apego a ambas figuras parentales predice el desarrollo de las competencias sociales de los jóvenes contando con la mediación de la relación con los pares (e.g., Mota, \& Matos, 2008). Recientemente Rocha, Mota y Matos (2011) en un estudio conducido con 742 jóvenes entre 12 y 23 años de edad, soportan el concepto de la autoestima en el role mediador en la asociación entre la calidad del apego a la madre y la calidad de desarrollo de relación con los pares. Algunos estudios sugieren que los jóvenes con buenos índices de confianza personal y ajuste saludable (Allen, Moore, Kuperminc \& Bell, 1998; Burge, et al., 1997; Paterson, Pryor \& Field, 1995), parecen reflejar una mayor facilidad en encarar e ultrapasar las dificultades. Particularmente en nuestro estudio no se ha confirmado el papel mediador de la relación con los pares entre el apego a los padres e la autoestima. Pensamos que talvez una justificación podrá ser que los pares puedan ejercer un role moderador más que mediador en esta asociación.

En resumen, el presente estudio ha señalado la importancia del papel que otras figuras significativas, como los pa- 
res y profesores, tienen en el desarrollo psicosocial de los adolescentes, especialmente en la autoestima y coping. Además, permitió entender el papel de mediación de la relación con los pares y la autoestima en la asociación entre la calidad del lazo emocional con los padres y el coping activo. Aún que no se hayan testado también consideramos pertinente el análisis del efecto moderador de las variables de relación con los pares y la autoestima, datos que deberían ser equacionados en el futuro. Pensamos que este estudio aporta datos relevantes que deben ser transpuestos para la práctica, especialmente en lo que concierne a la calidad de la relación con figuras significativas en el contexto escolar. Sabemos que la calidad de la relación con profesores y otras figuras de la escuela puede proteger los jóvenes del riesgo (interno y externo a si). Así mismo ya que los jóvenes permanecen parte significativa de su tiempo en la escuela es necesario apostar en la formación en contexto escolar por medio de que se cultive la proximidad y cooperación entre familia y escuela. Destacamos sobretodo una mayor participación de los padres y profesores en la construcción de proyectos educativos y adecuación de enseñanza de acuerdo con las necesidades personales de los jóvenes (académicas y emocionales). Otro punto importante de destaque podría centrarse en la formación de los profesores hacia competencias de gestión específicas. Así mismo el contexto escolar y la sala de aula representan una oportunidad de expresión de la diferencia, gestión de conflictos e negociación de puntos de vista e resolución de problemas de diversos órdenes.

\section{Referencias}

Ainsworth, M. D. S., Blehar, M., Waters, E., \& Wall, S. (1978). Patterns of attachment. Hillsdale, New Jersey: Lawrence Erlbam Associates.

Allen, J. P., MacElhaney, K. B., Land, D. J., Kupermic, G. P., Moore, C. W., O’Beeirne Kelly, H., et al. (2003). A secure base in adolescence: Markers of attachment security in the mother-adolescent relationship. Child Development, 74, 292-307.

Allen, J. P., Moore, C., Kuperminc, G., \& Bell, K. (1998). Attachment and adolescent psychosocial functioning. Child Development, 69, 1406-1419.

Al-Yagon, M., \& Mikulincer, M. (2006). Children's appraisal of teacher as a secure base and their socio-emotional and academic adjustment in middle childhood. Research in Education, 75, 1-18.

Armsden, G. C., \& Greenberg, M. T. (1987). The inventory of parent and peer attachment: individual differences and their relationship to psychological well-being in adolescence. Journal of Youth and Adolescence, 16, 427-454.

Arndt, J., \& Goldenberg, J. L. (2002). From threat to sweat: The role of physiological arousal in the motivation to maintain self-esteem. In A. Tesser, D.A. Stapel, \& J. V. Wood (Eds.), Self and motivation: Emerging psychological perspectives (pp. 43-69). Washington, DC: American Psychological Association.

Assor, A., \& Tal, K. (2012). When parents' affection depends on child's achievement: Parental conditional positive regard, self-aggrandizement, shame and coping in adolescents. Journal of Adolescence, 35, 249-260.

Bandalos, D. L., \& Finney, S. J. (2001). Item parceling issues in structural equation modeling. In G. A. Marcoulides \& R. E. Schumacker (Eds.), Advanced structural equation modeling: New developments and techniques. Mahwah, NJ: Lawrence Erlbaum Associates, Inc.

Baron, R. M., \& Kenny, D. A. (1986). The moderator-mediator variable distinction in social psychological research: Conceptual, strategic, and statistical considerations. Journal of Personality \& Social Psychology, 51, 11731182.
En este estudio también importa destacar algunas limitaciones que requieren precaución al generalizar los resultados. En suma, aunque el modelo se haya construido sobre sólidas bases teóricas, se trata de un estudio transversal, no permite una comparación de los resultados observados en el tiempo, por lo que la dirección de causalidades se supone. Por lo tanto, sería interesante utilizar los estudios longitudinales, a fin de observar el proceso de desarrollo implícito.

Como nota final, algunas orientaciones para la investigación futura podrían ir al encuentro de análisis de la calidad de las relaciones en diferentes configuraciones familiares (familias monoparentales, familias reconstituidas,...), también prestando atención a los adolescentes institucionalizados. Aunque no haya sido abordado en el presente estudio, sabemos que esta variable pude tener un efecto significativo en el desarrollo del apego parental, lo que es pasible de afectar la forma como los jóvenes se perciben a sí mismos e incluso como resuelven sus dificultades. Por último, enfatizamos que este estudio se basó en datos obtenidos a través del autoinforme, por lo que además sería relevante observar contribuciones de la calidad de la relación entre los jóvenes, entre otros, a través de la percepción de los padres y profesores.

Agradecimientos.- Trabajo financiado en el ámbito del proyecto de investigación de la Fundación para la Ciencia y Tecnología - Portugal (Refa. PTDC/PSI/65416/2006) y por fondos FEDER en el ámbito del Programa Operacional de Factores de Competitividad (POFC) _ COMPETE.
Benson, M. J., McWey, L. M., \& Ross, J. J. (2006). Parental Attachment and Peer Relations in Adolescence: A Meta-Analysis. Research in Human Development, 3, 33-43.

Beyers, W., \& Seiffge-Krenke, I. (2007). Are friends and romantic partners 'the best medicine'? How the quality of other close relations mediates the impact of changing family relationships on adjustment. International Journal of Behavioral Development, 31, 559-568.

Bollen, K.A. (1990). Overall fit in covariance structure models: Two types of sample size effects. Psychological Bulletin, 107, 256-259.

Bowlby, J. (1969). Attachment. London: Penguin.

Bowlby, J. (1973). Attachment and loss. (Vol. II: Separation). New York: Basic Books.

Bronfenbrenner, U. (1996). A ecologia do desenvolvimento bumano: Experimentos naturais e planejados. Porto Alegre: Artes Médicas.

Buhl, H. M. (2008). Significance of individuation in adult child-parent relationships. Journal of Family Issues, 29, 262-281.

Burge, D., Hammen, C., Davila, J., Daley, S., Paley, B., Hezberg, D., \& Lindberg, N. (1997). Attachment cognitions and college and work functioning two years later in late adolescent women. Journal of Youth and Adolescence, 26, 285-301.

Byrne, B. M. (1998). Structural equation modeling with LISREL, PRELIS, and SIMPLIS: Basic concepts, Applications, and programming. Erlbaum, Mahwah, NJ.

Cleto, P. M., \& Costa, M. E. (1995). Coping across situations questionnaire CASQ. Adaptação do instrumento (Manuscrito não publicado). Faculdade de Psicologia e de Ciências da Educação da Universidade do Porto.

Cohen, P., Kasen, S., Chen, H., Hartmark, C., \& Gordon, K. (2003). Variations in patterns of developmental transitions in the emerging adulthood period. Developmental Psychology, 39, 657-669. 
Davis, H. A. (2003). Conceptualizing the role and influence of studentteacher relationships on children's social and cognitive development. Educational Psychologist, 38, 207-234.

Deković, M., Wissink, I. B., \& Meijer, A. M. (2004).The role of family and peer relations in adolescent antisocial behaviour: comparison of four ethnic groups. Journal of Adolescence, 27, 497-514.

Drew, L. M., Berg, C, \& Wiebe, D. J. (2010). The mediating role of extreme peer orientation in the relationships between adolescent-parent relationship and diabetes management. Journal of Family Psychology, 24, 299306. doi: $10.1037 / \mathrm{a} 0019352$.

Dubois, D. L., Felner, R. D., Brand, S., Adan, A. M., \& Evans, E. G. (1992). A prospective study of life stress, social support, and adaptation in early adolescence. Child Development, 63, 542-557.

Eccles, J. S., \& Roeser, R. W. (2003). Schools as developmental contexts. In G. R. Adams \& M. Berzonsky (Eds.), Blackwell handbook of adolescence (pp. 129-148). Oxford: Blackwell Publishing.

Eccles, J. S., Templeton, J., Barber, B., \& Stone, M. (2003). Adolescence and emerging adulthood: the critical passage ways to adulthood. In M.H. Bornstein, L. Davidson, C. L. M. Keyes \& K. A. Moore (Eds.), Wellbeings: Positive development across the life course (pp.383-406). Mahwah, NJ: Lawrence Erlbaum Associates.

Engels, R. C., Dekovic, M., \& Meeus, W. (2002). Parenting practices, social skills and peer relationships in adolescence. Social and Behaviour Personality, 30, 3-18.

Ferreira, M., \& Costa, M. E. (1998). Inventory of peer and parental attachment. Adaptación del instrumento. Manuscripto no publicado. Faculdad de Psicologia y de Ciências de la Educación da Universidad do Porto.

Field, A. (2005). Discovering statistics using SPSS. $2^{\circ}$ Edition, London: Sage Publications.

Freeman, H., \& Brown, B. B. (2001). Primary attachment to parents and peers during adolescence: Differences by attachment style. Journal of Youth and Adolescence, 30, 653-675.

Gifford-Smith, M. E., \& Brownell, C. A. (2003) Childhood peer relationships: social acceptance, friendships, and peer networks. Journal of School Psychology, 41, 235-284.

Grossmann, K. E., \& Grossmann, K. (2004). Universality of human social attachment as an adaptive process. In C.S. Carter, L. Ahnert, K.E. Grossmann, S.B. Hrdy, M.E. Lamb, S.W. Porges, \& N. Sachser (Eds.), Attachment and bonding: A new synthesis (pp.199-229). Cambridge; MA: The MIT Press.

Guedeney, N., \& Guedeney, A. (2004). Vinculação: conceitos e aplicações. Lisboa: Climepsi Editores.

Hershenberg, R., Davila, J., Yoneda, A., Starr, L. R., Miller, M.R., Stroud, C. B., Feinstein, B. A. (2011). What I like about you: The association between adolescent attachment security and emotional behavior in a relationship promoting context. Journal of Adolescence, 34, 1017-1024.

Jiménez, T. I. (2011). Autoestima de riesgo y protección: Una mediación entre el clima familiar y el consumo de sustancias en adolescentes. Intervencion Psicosocial, 20, 53-61.

Laible, D. J., Carlo, G. \& Roesch, S. C. (2004). Pathways to self-esteem: The role of parent and peer attachment, sympathy, and social behaviors. Journal of Adolescence, 27, 703-716.

Larouse, S., Tarabulsy, G., \& Cyrenne, D. (2005). Perceived autonomy and relatedness as moderating the impact of teacher-student mentoring- relationship on student academic adjustment. The Journal of Primary Prevention, 26, 111-128.

Leary, M. R., \& Baumeister, R. F. (2000). The nature and function of selfesteem: Sociometer theory. In M. P. Zanna (Ed.). Advances in experimental social psychology. Vol. 32 (pp. 2-62). San Diego, CA: Academic Press.

Litlle, T. D.,Cunningham, W. A., Shahar, G., \& Widman, K. F. (2002). To parcel or not to parcel: Exploring the question, weighing the merits. Structural Equational Modeling, 9, 151-173.

Llinares Insa, L. I., Molpeceres Pastor, M. A. \& Musitu Ochoa, G. (2001). La autoestima y las prioridades personales de valor. Un análisis de sus interrelaciones en la adolescencia. Anales de Psicología, 17, 189-200.

MacCallum, R. C., Widaman, K. F., Preacher, K. J., \& Hong, S. (2001). Sample size in factor analyses: The role of model error. Multivariate Behavioral Research, 36, 611-637.

Maroco, J. (2007). Análise estatística com utilização do SPSS. Lisboa: Sílabo.
McLean, K. C., \& Jennings, L. E. (2012). Teens telling tales: How maternal and peer audiences support narrative identity development. Journal of Adolescence, 35, 1455-1469

Matos, P. M. (2003). O conflito à luz da teoria da vinculação. In M. E. Costa (Coord.), Gestão de conflitos na escola (pp.144-191). Lisboa: Universidade Aberta.

Matos, M. P. \& Costa, E. M. (2001). Cuestionario de apego al padre y la madre. (Manucripto no publicado). Facultad de Psicologia y Ciências de la Educación. Universidad do Porto.

Gouveia, T., \& Matos, P. M. (2011). Manual do Ouestionário de Vinculacão ao Pai e à Mãe (QVPM). Facultad de Psicologia y Ciências de la Educación. Universidad do Porto. Recuperado de https://sites.google.com/site/manualqvpm

Meeus, W., Iedema, J., Maassen, G., \& Engels, R. (2005). Separationindividuation revisited: On the interplay of parent-adolescent relations, identity and emotional adjustment in adolescence. Journal of Adolescence, 28, 89-106.

Meeus, W., Oosterwegel, A., \& Vollebergh, W. (2002). Parental and peer attachment and identity development in adolescence. Journal of Adolescence, 25, 93-106.

Mikami, A. Y., Gregory, A., Allen, J. P., Pianta, R. C., \& Lun, J. (2011). Effects of a teacher professional development intervention on peer relationships in secondary classrooms. School Psychology Review, 40, 367-385.

Millings, A., Buck, R., Montgomery, A., Spears, M., Stallard, P. (2012). School connectedness, peer attachment, and self-esteem as predictors of adolescent depression. Journal of Adolescence, 35, 1061-1067.

Mota, C. P., \& Matos, P. M. (2005). Questionário de ligacão a professores e funcionários - QLPF (Manuscrito no publicado). Facultad de Psicologia y de Ciências de la Educación de la Universidade do Porto.

Mota, C.P., \& Matos, P.M. (2006). Propriedades psicométricas do coping across situations questionnaire - CASQ numa amostra de adolescentes portugueses. Psychologica, 43, 211-226.

Mota, C. P., \& Matos, P. M. (2008). Competências sociais e variáveis relacionais em adolescentes. Revista Psicologia, Educação e Cultura, 12, 61-86.

Mota, C. P., \& Matos, P. M. (2009). Vinculação, conflito e auto-estima em adolescentes de famílias intactas e divorciadas. Psicologia: Reflexão e Crítica, 22, 317-325.

Nickerson, A. B., \& Nagle, R. J. (2005). Parent and peer attachment in late childhood and early adolescence. Journal of Early Adolescence, 25, 223-249.

Pallant, J. (2001). SPSS survival manual. Buckingham: Open University Press.

Paterson, J., Pryor, J., \& Field, J. (1995). Adolescent attachment to parents in relation to competence, depression, and anxiety: A longitudinal study. Journal of Early Adolescence, 12, 420-440.

Pianta, R. C. (1999). Enhancing relationships between children and teachers. Washington DC: American Psychological Association.

Pianta, R. C., Steinberg, M. S., \& Rollins, K. B. (1995). The first two years of school: Teacher-child relationships and deflections in children's classroom adjustment. Development and Psychopathology, 7, 295-312.

Preacher, K. J., \& Hayes, A. F. (2004). SPSS and SAS procedures for estimating indirect effects in simple mediation models. Behavior Research Methods, Instruments, \& Computers, 4, 717-731.

Rhodes, J. E., Grossman, J. B., \& Resch, N. L. (2000). Agents of change: Pathways through which mentoring relationships influence adolescents' academic adjustment. Child Development, 71, 1662-1671.

Riley, P. (2011). Attachment theory and the teacher-student relationship. $1^{\text {st }}$ Edition. Oxon: Routdlege.

Robins, R. W., \& Trzesniewski, K. H. (2005). Self-esteem development across the lifespan. American Psychological Society, 3, 158-162.

Rocha, M. (2008). O desenvolvimento das relações de vinculação na adolescência: Associações entre contextos relacionais com os pais, pares e par amoroso (Dissertación presentada para la obtención del Grado de Doctor) .Universidad do Porto, Faculdad de Psicologia y de Ciências da Educación

Rocha, M., \& Matos, P. M. (2003). Rosenberg's self-esteem scale. Adaptação para a população portuguesa. Manuscripto no publicado. Faculdad de Psicología y de Ciências de la Educación de la Universidad do Porto.

Rocha, M., Mota, C.P., \& Matos, P. M., (2011). Vinculação à mãe e ligação aos pares na adolescência: $\mathrm{O}$ papel mediador da auto-estima. Análise Psicológica, 2, 185-200.

Rosenberg, M. (1965). Society and the adolescent self-image. Princeton, N.J.: Princeton University Press. 
Seiffge-Krenke, I. (1995). Stress, coping and relationships in adolescence. New Jersey: LEA Books.

Seiffge-Krenke, I., \& Shulman, S. (1990). Coping style in adolescence: A cross-cultural study. Journal of Cross-Cultural Psychology, 21, 351-377.

Shipley, B. (1999). Testing causal causal explanations in organismal biology: causation, correlation and structural equation modelling. Oikos, 86, 374382.

Soenens, B., \& Vansteenkiste, M. (2005). Antecedents and outcomes of selfdetermination in three life domains: The role of parents' and teachers' autonomy support. Journal of Youth and Adolescence, 34, 589-604.

Verschueren, K., Doumen, S., Buyse, E. (2012). Relationships with mother, teacher, and peers: Unique and joint effects on young children's selfconcept. Attachment \& Human Development, 14, 233-248.
Wentzel, K. R. (2002). Are effective teachers like good parents? Teaching styles and student adjustment in early adolescence. Child Development, 73 , 287-301.

Wilkinson, R. B. (2010). Best friend attachment versus peer attachment in the prediction of adolescent psychological adjustment. Journal of Adolescence, 33, 709-717.

Yuan, K.H. (2005). Fit indices versus test statistics. Multivariate Behavioral Re search, 40, 115-148.

Zimmer-Gembeck, M. J., \& Locke, E. M. (2007).The socialization of adolescent coping behaviours: Relationships with families and teachers. Jour nal of Adolescence, 30, 1-16.

(Artículo recibido: 25-10-2012; revisado: 13-2-2012; aceptado: 18-2-2013) 